\title{
Circadian Timing: From Genetics to Behavior
}

\author{
Patricia V. Agostino, Ivana L. Bussi and Carlos S. Caldart
}

\section{1 \\ General Aspects of Biological Timing}

In order to adapt to a changing environment, most living organisms have established the ability to measure time by the development of biological timing systems. There are different time-scales in which these temporal systems operate, thus covering more than 12 orders of magnitude, from microseconds to days or even seasons. All of these biological 'clocks' use time-keeping mechanisms that provide organisms with temporal information crucial to fulfill the required biological processes (Buhusi \& Meck, 2005; Golombek, Bussi, \& Agostino, 2014). Most chapters in this book are focused on temporal processing in the millisecond-to-minutes range, which is crucial to many forms of cognitive processing. In the present chapter, we will cover different aspects of biological timing in the circadian (i.e., 'about a day', 24-h) range. Since circadian timing is ubiquitous and regulates many physiological and behavioral functions - including cognitive functions - the study of circadian rhythms has a deep impact in neuroscience, medicine and health research.

Despite early classical experiments such as De Mairan's 1729 demonstration of an endogenous rhythm in leaf movement of Mimosa plants, among many others, the notion of an inner biological clock remained quite elusive until the mid-twentieth century (Golombek \& Rosenstein, 2010). Several lines of evidence revealed the presence of an endogenous daily biological clock, and the term circadian was introduced in the fifties to identify self-sustained rhythms under constant conditions.

At present, it is very well known that the circadian clock is an endogenous timing system that organizes the environmental oscillations every $24-\mathrm{h}$. The periodic changes in the light/dark cycle produced by the rotation of the Earth influenced the development of circadian rhythms in almost all living organisms. Hence, organisms are able to adapt to and anticipate the changes in lighting conditions, thus settling their physiological processes to specific times of the day. Some physiological and behavioral functions regulated by the circadian system include the sleep-wake cycle, body temperature, hormone release, and gene expression. For example, nearly half of all mammalian genes are rhythmically expressed in one or more tissues (Yan, Wang, Liu, \& Shao, 2008). 
The circadian system have three fundamental characteristics: (1) the presence of an endogenous oscillator, which generates rhythms with a period close to 24 -h in the absence of external cues; (2) the capacity to be synchronized by rhythmic environmental stimuli; and (3) temperature compensation, which means that the biochemical reactions that take place in the circadian clock do not change their speed with temperature variations (Buhr \& Takahashi, 2013). Additionally, the circadian system is composed by three main components that allow the regulation of physiology and behavior: (1) an input pathway sensing the environment, (2) an endogenous core oscillator, and (3) an output pathway that couples the core oscillator with the rest of the organism. In mammals, the main circadian oscillator (called the core or master oscillator) is located in the suprachiasmatic nuclei (SCN) of the ventral hypothalamus. The SCN synchronizes subordinate organ and tissue clocks (called peripheral circadian oscillators) using mainly autonomic pathways from the hypothalamus, driving neurochemical, endocrine, and metabolic signaling pathways (Albrecht, 2012).

\section{Molecular Components of the Circadian System}

The SCN neurons have the ability to generate autonomous circadian rhythms (Welsh, Logothetis, Meister, \& Reppert, 1995). The intrinsic rhythmicity of each neuron is based on a molecular oscillation that involves negative feedback loops of transcriptional and translational processes (Figure 1.1). In the mammalian circadian system, a primary feedback loop is composed by the positive elements CLOCK and BMAL1, which heterodimerize (i.e., join themselves to form a complex of non-identical monomers) and promote the transcription of Per (Period) and Cry (Cryptochrome) genes (negative elements) by acting on specific biding sites (E-box sequences) located in their promoter region (Gekakis et al., 1998; Munoz, Brewer, \& Baler, 2006; Yoo et al., 2005). After transcription and translation, PER and CRY proteins heterodimerize and translocate to the nucleus to inhibit their own transcription by acting on the dimer CLOCK/BMAL1 (Ye et al., 2014).

Each transcriptional/translational loop takes near to 24-h to complete. In addition to this primary loop, another negative feedback loop contributes to clock precision and robustness. In the second loop, the protein REV-ERB $\alpha$ moves to the nucleus to repress Bmaly expression; inversely, Ror $\alpha$ can stimulate its transcription, both binding to RORE (Retinoic acid-related Orphan receptor Response Element) sites on the Bmalt promoter (Guillaumond, Dardente, Giguere, \& Cermakian, 2005; Preitner et al., 2002; Sato et al., 2004). These oscillations of negative and positive elements generate circadian rhythmicity, 


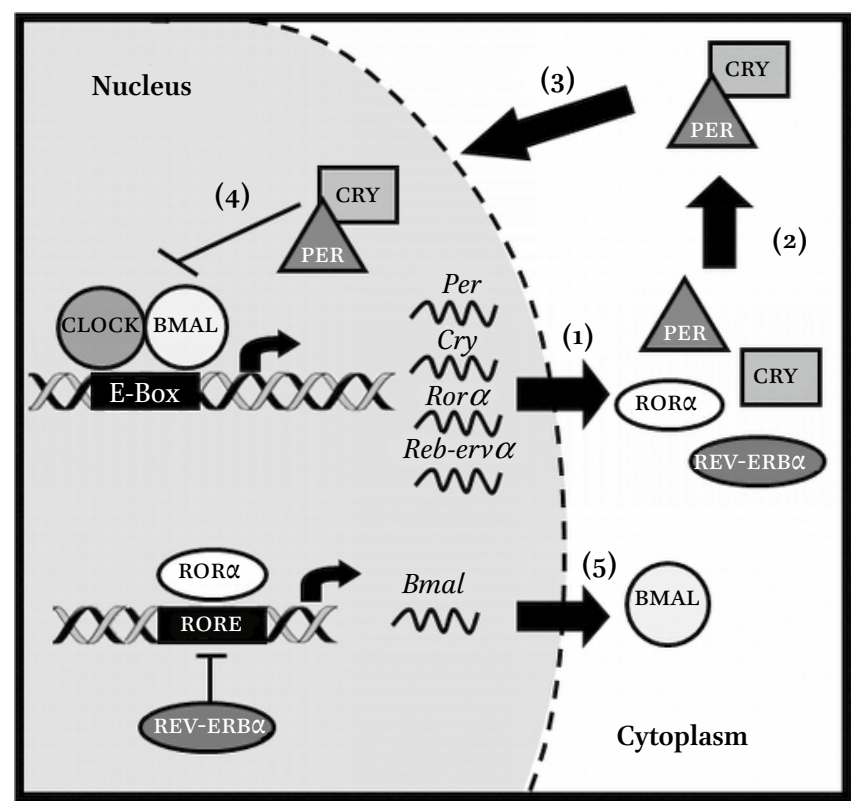

FIGURE 1.1 The molecular circadian clock. Simplified model of the transcriptional/translational feedback loops that constitute the mammalian circadian clock. In the primary feedback loop, the positive elements CLOCK and BMALr initiate transcription of target genes containing E-box sequences (1), including Period (in mice, Per1, Per2, and Per3) and Cryptochrome (Cryı and Cry2). Once in the cytoplasm, the resulting PER and CRY proteins heterodimerize (2) and translocate back to the nucleus (3). Negative feedback is achieved by PER:CRY heterodimers to repress their own transcription by acting on the CLOCK:BMALi complex (4). Another regulatory loop is induced by CLOCK:BMALi heterodimers activating transcription of Rev-erb $\alpha$ and Ror $\alpha$. REV-ERB $\alpha$ and ROR $\alpha$ subsequently compete to bind RORE elements present in Bmalı promoter (5). Thus, the circadian oscillation of Bmalı is both positively and negatively regulated by ROR $\alpha$ and REV-ERB $\alpha$.

which regulates the circadian output pathway by driving downstream clockcontrolled gene (CCG) expression. The regulation of CCGs is thought to be tissue-specific. Indeed, recent studies on mouse tissues indicate that roughly $50 \%$ of all genes oscillate with a circadian periodicity (Zhang, Lahens, Ballance, Hughes, \& Hogenesch, 2014). Similar auto-regulatory feedback loops were also described for other organisms, including Drosophila, zebrafish, and cyanobacteria (Bell-Pedersen et al., 2005). Notably, the circuits that generate molecular oscillations of circadian clock genes are remarkably conserved 
among species, suggesting a possibly monophyletic origin of such mechanism (Dunlap, Loros, \& DeCoursey, 2004).

The circadian system in nature is normally exposed to a rhythmic 24-h environment, so that appropriate signals (called Zeitgebers, from German Zeit, 'time'; geben, 'to give'), such as light, temperature, or food, synchronize its oscillation (Golombek \& Rosenstein, 2010). This effect in Chronobiology is called entrainment. Therefore, under entrained conditions, circadian rhythms adjust their endogenous period (called $\tau$ ) to that of the Zeitgeber (called T). It is worth to mention that the concept of entrainment - the phenomenon in which two or more independent rhythmic processes synchronize with each other - has been described in a wide variety of systems and over a wide range of timescales: some examples include fireflies illuminating in synchrony, human individuals adjusting their speech rhythms to match each other in conversation, foot tapping to the beat of a song, and attention to rhythmic patterns in the milliseconds scale (Jones, 1976; Clayton, Sager, \& Will, 2005). Circadian rhythms of living organisms are entrained to rhythmic environmental cues, such as the 24-h light/dark alternation or daily temperature cycles. Locomotor activity rhythms are one of the most studied circadian outputs, and are frequently represented as actograms (Figure 1.2), where horizontal lines represent consecutive days and black bars describe the locomotor activity episode. Each horizontal line in Figure 1.2 shows one experimental day along the vertical axis, and the hours of the day are represented in the $\mathrm{x}$ axis. Further information about actograms is provided in Section 6.3.

In mammals, the most powerful synchronizer is the daily light/dark cycle. Under normal 24-h light/dark conditions, circadian rhythms are entrained with a period equal to 24 -h (Figure 1.2A).

Light stimulates a group of photosensitive retinal ganglion cells (pRGC) that express the photopigment melanopsin (Panda et al., 2002) and project to the SCN through the retinohypothalamic tract (Morin, 2013). The effect of light on SCN neurons leads to an increase in the intracellular concentrations of $\mathrm{Ca}^{2+}$, which initiates a signal transduction cascade that ultimately results in a phase shift of the circadian clock (Golombek, Agostino, Plano, \& Ferreyra, 2004; Golombek et al., 2003; Golombek \& Rosenstein, 2010; Morin \& Allen, 2006). The circadian clock differs in its temporal responsiveness to external stimuli. For example, in nocturnal rodents, exposure to light synchronizes circadian rhythms by inducing phase delays during the early night and phase advances 
(A)

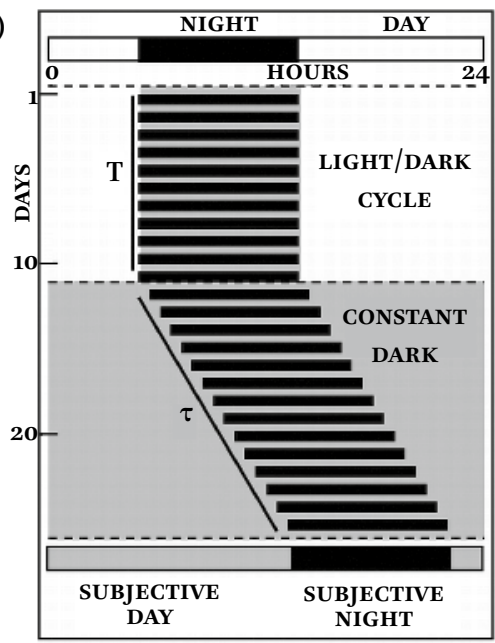

(B)

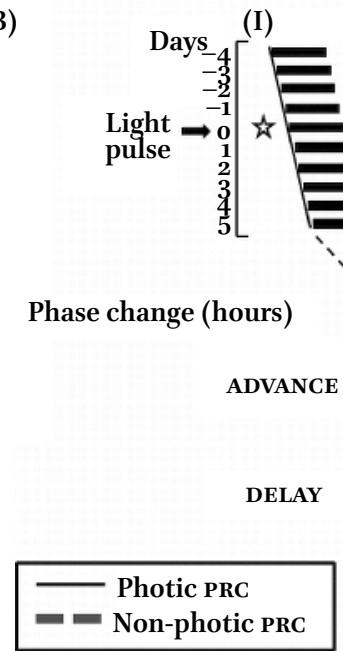

(II)

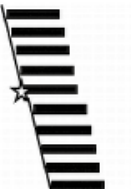

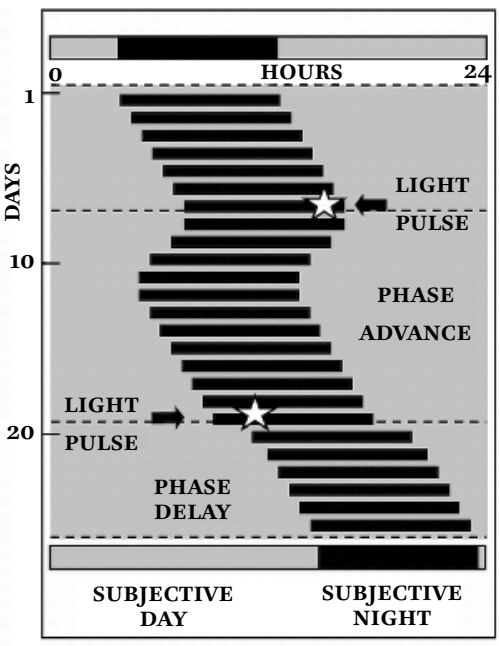

(III)
(IV)

(V)
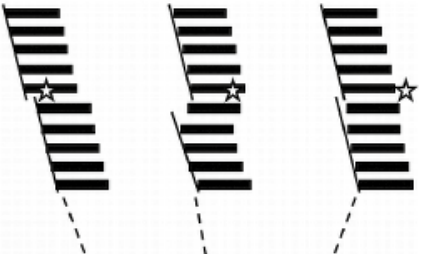

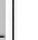


during the late night (Figure 1.2A), led by divergent signal transduction pathways that ultimately drive the activation of circadian clock genes (Lowrey \& Takahashi, 2000). For example, during the late night, when light induces phase advances of behavioral rhythms, photic stimulation specifically activates the guanylyl cyclase (GC)/cGMP/cGMP-dependent kinase (PKG) pathway (Agostino, Plano, \& Golombek, 2007; Golombek et al., 2004).

The effect of light in synchronizing the circadian clock depends on the time of day, thus defining a phase-response curve (PRC) that indicates the times at which light can induce phase delays, phase advances, or no change at all (Figure 1.2B, Dunlap et al., 2004; Golombek \& Rosenstein, 2010; Johnson, Elliott, \& Foster, 2003). The PRC is one of the main tools to study sensitivity of the circadian system to either light (photic entrainment) or many other stimuli potentially capable of resetting the clock (called non-photic entrainment). The PRC, its shape and amplitude, is an intrinsic property of the circadian oscillator. Indeed, the PRC is characteristic for each species. In humans, it is also the basis for the design of treatments in abnormal entrainment situations, such as jet-lag, shiftwork or circadian-related sleep disruption (Gooley, 2008; Lewy, Emens, Jackman, \& Yuhas, 2006).

Other entrainment cues like food intake, environmental changes in temperature, drugs or even social interactions can reset circadian rhythms, eliciting phase shifts mainly during the subjective day (the resting period in rodents; Figure 1.2B).

When photic or non-photic Zeitgebers directly affect a rhythmic output without affecting the circadian oscillator, for instance directly suppressing or stimulating locomotor activity, this mechanism is called masking (Mrosovsky, 1999). Different from entrainment, masking influences a circadian output (such as behavior) without the participation of the endogenous circadian clock.

\subsection{Zeitgeber Time and Circadian Time}

Since a strong Zeitgeber defines the rhythm of the circadian oscillator, under entrained conditions time is expressed as Zeitgeber time (zT). In the case of photic entrainment, within a light/dark schedule of 12 hours of light and 12 hours of darkness (LD 12:12), ZTo is defined as the time of 'lights ON' (i.e., the beginning of the light phase). Similarly, ZT12 corresponds to the time of 'lights OFF', the end of the light phase. Analogous terminology is applied for non-photic Zeitgebers, such as food or temperature.

When organisms are kept under constant conditions by avoiding contact with external time cues, they display so-called freerunning or circadian rhythms, no longer equal to 24-h. Therefore, time cannot be expressed in ZT but it is expressed in circadian time (СT) units. One circadian cycle is divided 
into 24 CTs, with one unit being defined as the division of the internal period length $(\tau)$ by 24 hours. The period under freerunning conditions differs among species. For example, the freerunning period of the human circadian rhythm in body temperature is close to 25 -h.

\subsection{In the Limit of Synchronization: T-cycles, Forced Desynchronization and Relative Coordination}

Under natural conditions of entrainment to a Zeitgeber of $\mathrm{T}=24-\mathrm{h}$, variations of the subject's endogenous period $(\tau)$ may lead to different phases of entrainment, that are associated with chronotypes. In this sense, short endogenous periods $\tau$ often lead to early phases ('morning larks') and long periods $\tau$ correspond to late phases ('night owls'). Mutations that affect the intrinsic period $\tau$ result in large shifts of the entrainment phase. For example, patients with familial advanced sleep phase syndrome have a short endogenous period of $\tau=23 \cdot 3^{-h}$ leading to a phase advance of more than 3 -h (Jones et al., 1999). Thus, the phase of entrainment is a function of the period mismatch $\tau-\mathrm{T}$ (Bordyugov et al., 2015).

Most organisms can only entrain to Zeitgebers cycling with a period close to 24 -h. If the entraining period is too short or too long - thus, exceeding the range of entrainment - the circadian system cannot follow the Zeitgeber anymore. In the laboratory, circadian rhythms can be entrained to variable Zeitgebers with different T periods (called T-cycles) in order to determine the range of entrainment for each species (Aschoff, Daan, \& Honma, 1982; Jud, Schmutz, Hampp, Oster, \& Albrecht, 2005). To determine this range of entrainment, T-cycles of different periods $\mathrm{T} \neq 24$-h are applied. For example, 22-h or 26-h T-cycles may be used to define the limits of stable entrainment. These limits differ from species to species and depend on the nature of the applied Zeitgeber. Notably, under T periods close to the limits of circadian entrainment, nocturnal rodents present two patterns of behavior, called forced desynchronization and relative coordination (Campuzano, Vilaplana, Cambras, \& Diez-Noguera, 1998; Casiraghi, Oda, Chiesa, Friesen, \& Golombek, 2012; de la Iglesia, Cambras, Schwartz, \& Diez-Noguera, 2004; Golombek et al., 2013). Forced desynchronization (Figure 1.3A) occurs when a system of dual, weakly coupled circadian oscillators is subjected to a Zeitgeber that is outside the range of entrainment of one of these oscillators. For example, it has been described that when rats were maintained under light/dark cycles with period of 22- and 23-h, two circadian rhythms were simultaneously present in their motor activity, temperature, and feeding activity: one rhythm followed the external light/dark cycle, whereas the other rhythm was under freerunning with a period of around 25-h (Madrid, Lax, Vilaplana, Cambras, \& Díez-Noguera, 1992; 
Vilaplana, Cambras, \& Diez-Noguera, 1997). This behavior has been found as the manifestation of two groups of oscillators within the $\mathrm{SCN}$ : a ventrolateral SCN capable of entraining to the external cycle and a dorsomedial SCN incapable of such kind of entrainment (de la Iglesia, Cambras, Schwartz, \& DiezNoguera, 2004). Therefore, under certain external conditions, the circadian system can be dissociated in such a way that an entrained and a non-entrained rhythm (black bars and white bars, respectively, in Figure 1.3A) could appear simultaneously. On the other hand, relative coordination (Figure 1.3B) reflects the resetting effects of environmental Zeitgebers that are strong enough to influence the biological clock but are not strong enough to synchronize it (Golombek et al., 2013).

\subsection{Circadian Desynchronization}

When the timing of the light/dark cycle is altered - for example, as a result of transmeridian travel or work schedules - it leads to a misalignment of the circadian system with the environment.

A well-known consequence of circadian alteration is jet-lag disorder, which results from rapidly crossing times zones - with the severity of symptoms typically depending on the number of times zones crossed, the travel frequency, and the direction of travel (east or west). Symptoms vary from insomnia and excessive sleepiness to more severe complications, including increased cancer risk, metabolic disorder, cognitive deficits, reduced fertility, and deregulated immune responses (Castanon-Cervantes et al., 2010; Golombek et al., 2013; Karatsoreos, Bhagat, Bloss, Morrison, \& McEwen, 2011; Mahoney, 2010; Scheer, Hilton, Mantzoros, \& Shea, 2009). In animal models, interference with the cGMP-related photic entrainment pathway has been proven to be a useful tool for accelerating synchronization to advances of the light/dark cycle, which may reduce jet-lag symptoms due to eastbound flights (Agostino et al., 2007; Plano, Agostino, de la Iglesia, \& Golombek, 2012).

Another circadian alteration is produced by shift work disorder, which affects people who frequently rotate shifts or work at night. Under these conditions, the alternation between a normal sleep/wake cycle and being awake at night and sleeping during the day not only alters the light/dark schedule in relation to the endogenous circadian clock, but also modifies feeding patterns, social habits, etc. (Reid \& Abbott, 2015; Zee \& Goldstein, 2010). Sleep loss, in addition to circadian misalignment, contributes to decreased alertness during night work. Current treatment of shift work disorder includes strategies to achieve and maintain some degree of circadian alignment (e.g., photic phase shift protocols, avoidance of light during the day, melatonin administration, etc.), improve sleep (using hypnotics, melatonin, and behavioral 
(A)

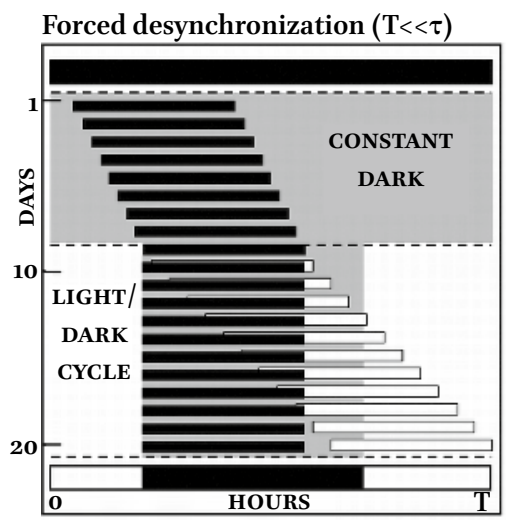

(B)

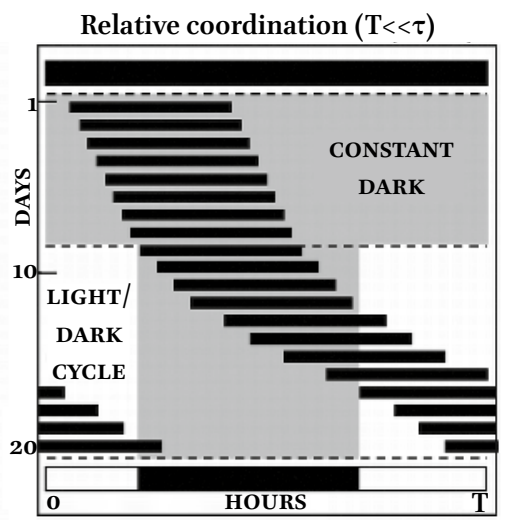

(C)

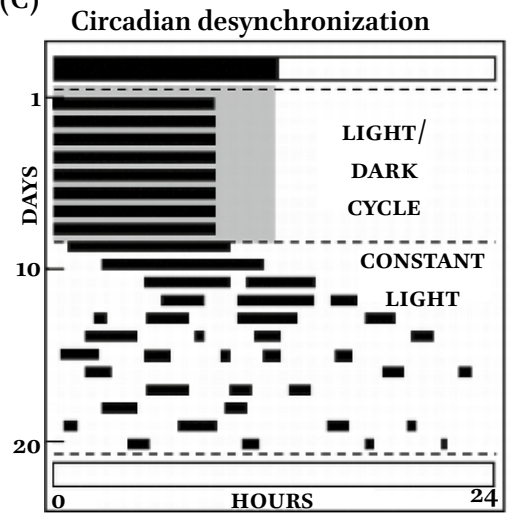

FIGURE 1.3 In the limit of circadian entrainment. Actograms representing different light/dark conditions in a nocturnal rodent. (A) When a T-cycle is outside the synchronization range (in this example, $T<<\tau)$ it leads to forced desynchronization. A dissociation of the behavioral rhythm into two components can be observed, one that is synchronized to the light/dark schedule (black bars), and a second one (white bars) that runs in relative coordination. (B) Relative coordination (modulation of the circadian period). $(C)$ Circadian desynchronization under constant light $(L L)$ conditions.

approaches), and facilitate alertness (using light, wake-promoting agents, and sleep scheduling).

Rodent models based on disruption of circadian rhythms provide useful information to study desynchronization due to modifications in the environment, such as abrupt changes in the light/dark cycle or nocturnal exposure to light. A commonly used model of circadian disruption is constant light $(\mathrm{LL})$ exposure (Figure 1.3C), which induces period lengthening followed by 
circadian arrhythmicity in nocturnal rodents (Meng et al., 2010; Moriya et al., 2000). Also, LL conditions attenuate circadian rhythms of clock genes such as Per2 (Bussi, Levin, Golombek, \& Agostino, 2014; Sudo et al., 2003). Altered circadian rhythms in rodents also lead to depressive-like symptoms, anxiety and anhedonia, expressed by reduced sucrose intake, altered patterns of food intake and high plasma levels of corticosterone (Fonken et al., 2009; Fonken et al., 2010; Tapia-Osorio, Salgado-Delgado, Angeles-Castellanos, \& Escobar, 2013), indicating that entrainment of circadian rhythms is necessary for efficient physiological and behavioral integrity.

\section{Circadian Influence on Cognition}

As mentioned, the circadian system regulates several physiological and behavioral functions, and disruption of the circadian clock may have severe consequences for physical and mental health. In this section, we will overview an important example of circadian modulation: the influence of the circadian system on cognitive functions.

There is evidence that cognitive performance and learning is influenced by circadian oscillators (Eckel-Mahan \& Storm, 2009; Gerstner \& Yin, 2010). Also, regularly-timed cognitive processes impact circadian rhythms (Gritton et al., 2012), indicating a bidirectional interaction between cognitive performance and circadian processes. Furthermore, at least for some tasks in nocturnal rodents, night-phase performance can never be equaled by light-phase performance regardless of the strength of entrainment to the schedule, which may have deep implications for shiftwork therapies (Gritton et al., 2012). In addition, desynchrony between internal and environmental time has been associated with impaired cognitive function in animals (Devan et al., 2001; Gibson, Wang, Tjho, Khattar, \& Kriegsfeld, 2010; Loh et al., 2010) and humans (Cho, 2001; Cho, Ennaceur, Cole, \& Suh, 200o; Folkard, 1996; Santhi, Horowitz, Duffy, \& Czeisler, 2007). Although it is well established that cognitive abilities vary as a function of daytime, there is still a widespread view that the circadian clock has a singular role in cognition related to sleep timing, and that the main factor for cognitive maintenance is the quality and duration of sleep. However, there is also evidence that indicates a circadian control of cognition beyond sleep timing, as revealed by forced desynchronization protocols (Kyriacou \& Hastings, 2010; Wright, Hull, \& Czeisler, 2002).

A fundamental component of cognition is the perception of the passage of time. In particular, temporal processing within the hundreds of milliseconds and the seconds-to-minutes range - known as interval timing - is crucial for many complex behaviors, such as speech comprehension, working memory, 
and decision making (Buhusi \& Meck, 2005; Agostino \& Cheng, 2016). Several evidences suggest that interval timing is also influenced by the circadian system. Indeed, time-of-day effects have been observed for the timing of both auditory and visual signals in the seconds-to-minutes range (Aschoff, 1985; Chandrashekaran et al., 1991; Meck, 1991; Pati \& Gupta, 1994). For example, several studies have reported that time judgments in humans covary with normal circadian rhythms (Kuriyama et al., 2005; Lustig \& Meck, 2001). Consistent with this finding, a circadian rhythm in interval timing was documented in control participants, but it was found to be disrupted in shift-workers (Pati \& Gupta, 1994). Moreover, rats exhibit circadian variations in time perception similar to those that have been demonstrated in humans (Shurtleff, Raslear, \& Simmons, 1990). Furthermore, sleep deprivation influences diurnal variation of interval timing in humans (Soshi et al., 2010). In Drosophila melanogaster, timing of short intervals is disrupted in circadian mutants for each of the three allelic per mutations, pers $^{\text {, }}$, er $^{l}$, and per $^{\circ}$ (Kyriacou \& Hall, 1980). In mice, significant differences in the estimation of 24-sec intervals at different times of day were reported (Agostino, do Nascimento, Bussi, Eguia, \& Golombek, 2011). These differences were maintained under constant dark conditions, but impaired in mice under constant light, which abolish circadian rhythmicity. Moreover, short time estimation in animals subjected to a 6-h advance of the light/dark cycle was transiently affected, indicating that temporal desynchronization of the circadian system is able to negatively affect interval timing. It has also been described that dopamine levels in mice present 24-h rhythms in the dorsal striatum, with lower levels during the day and peaking during the night (Bussi et al., 2014). Moreover, higher dorsal striatal dopamine levels during the night coincide with better performance on interval timing (i.e., peak location closer to the target time, higher peak amplitude and reduced peak width) in the nocturnal phase of the light/dark cycle in mice (Agostino et al., 2011; Bussi et al., 2014). Both interval timing and dopamine oscillation - as well as dopamine synthesis and turnover - in the dorsal striatum are affected by inducing circadian disruption under constant light conditions. In addition, circadian regulatory elements have been found in the promoter region of components involved in dopamine metabolism, like dopamine transporter (DAT), dopamine receptor $1 \mathrm{~A}(\mathrm{DRD} 1 \mathrm{~A})$, tyrosine hydroxylase $(\mathrm{TH})$, and monoamine oxidase (MAO), demonstrating that the expression of these components is under circadian regulation (Hampp et al., 2008). It was recently reported impaired learning in a timing task in pinealectomized rats (Bussi, Levin, Golombek, \& Agostino, 2015). Furthermore, melatonin depletion increased striatal dopamine availability - which was reversed by external melatonin administration - indicating that this hormone may modulate interval timing on a circadian base. 
Several outputs are used to assess the overall status of the circadian system. These variables (called circadian marker rhythms) can be easily measurable over long periods of time, preferably using non-invasive methods. The most widely used circadian marker rhythms in several organisms are those of activity, temperature, hormones, and clock gene expression (LeSauter \& Silver, 1998). In humans, the main variables used are actigraphy, core body temperature (Свт), and plasma or salivary melatonin (Bonmati-Carrion et al., 2014; Klerman, Gershengorn, Duffy, \& Kronauer, 2002). In nocturnal rodents, wheel-running activity is the most commonly used and reliable method for assessing the output of the circadian clock. Other measures that can be used include general activity, food-bin approaches, drinking, sleep, and body temperature.

Bioluminiscence has become a powerful tool for studying circadian rhythms. Exogenous luciferase genes, under the control of promoters conferring circadian regulation, have been introduced into a wide variety of organisms, including cyanobacteria (Synechococcus), plants (Arabidopsis), insects (Drosophila), nematodes (C. elegans) and rodents (Welsh \& Kay, 2005; Goya, Romanowski, Caldart, Bénard, \& Golombek, 2016). These transgenic organisms exhibit robust circadian rhythms of luminescence, useful as a longitudinal assay of rhythmicity for a wide range of recent genetic and biochemical studies of circadian clock mechanisms.

\section{Circadian Data Analysis}

\subsection{Period, Amplitude and Phase}

Like other periodic patterns, circadian rhythms can be described using mainly three parameters: a) period $(\tau)$, defined as the time in which a cycle is completed, b) amplitude, defined as the difference between the peak (or trough) and the mean value of the variable under study, and c) phase or phase angle $(\varphi)$, the timing of a reference point in the cycle relative to a fixed event (Figure 1.4). There are several conventions to define the phase of a rhythm; however, the most commonly used is when the amplitude reaches its maximum, which is called acrophase in the Cosinor analysis (see below; Dunlap et al., 2004). Consequently, every time that we mention the phase of a rhythmic signal, it will be referring to its acrophase. Indeed, the algorithms that will be described in the following sections will also compute the acrophase (time of peak) of the signal. 


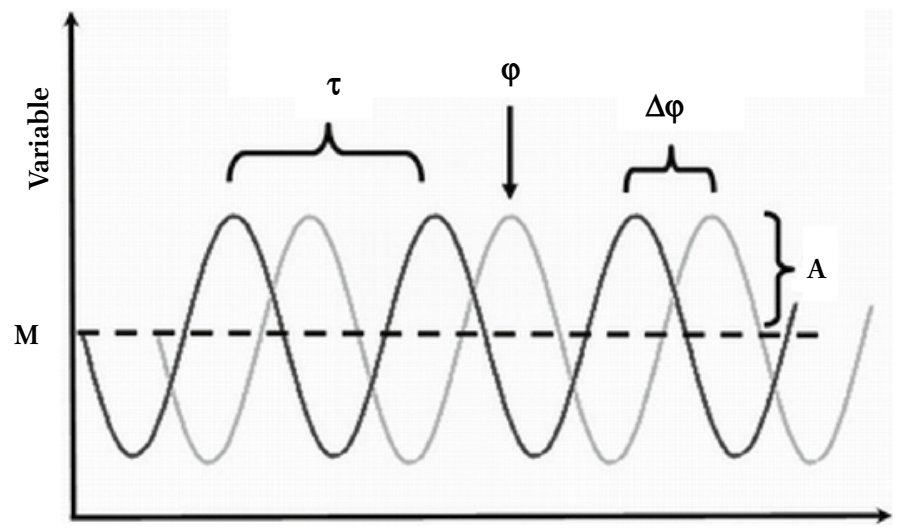

Time

FIGURE 1.4 Characteristics of circadian rhythms. Period $(\tau)$ is defined as the time to complete a cycle. It is commonly measured from peak to peak, but it could equally be measured from trough to trough or from any specified phase marker. The amplitude (A) of the rhythm is defined as one-half the peak-to-trough distance, from mesor $(M)$ to peak or $M$ to trough. Phase or phase angle $(\phi)$ is the timing of a reference point in the cycle (e.g., the peak) relative to a fixed event. The relationship of two mutually synchronized or entrained rhythms is expressed in terms of the phase angle difference $(\Delta \phi)$.

These parameters (period, amplitude, and phase) can describe any periodic pattern, but are normally used to characterize a sinusoidal wave. In many cases, however, this is not the real situation (i.e., locomotor activity may behave as a square-like rhythm). For this reason, most of the algorithms that are usually used to compute period and phase have been tested for different waveforms and are still valid for data analysis. Even though in this chapter we will only analyze the mentioned parameters, it is worth noting that according to the type of experimental data there are many other parameters that can be useful to analyze (e.g., sinusoidal dampening, fragmentation, coupling of the activity and Zeitgeiber, phase angle, etc.).

\subsection{Sampling and Signal Conditioning}

Before a complete overview of the methods used for analyzing circadian rhythms, it is important to refresh some basic concepts of signal analysis, such as sampling and signal conditioning (filtering and detrending). This information will allow us to know beforehand some of the limitations of our analytic methods. 
A fundamental aspect of data analysis in temporal series is sampling. The first step is to decide between uniform sampling and non-uniform sampling (i.e., when data cannot be collected uniformly spaced). When possible, a uniform sample is clearly the best option, because it allows a wider and stronger set of statistical tools. Even if some data points were lost, the missing data could still be interpolated in a reliable and easier way. Another key feature of the experimental design is to define the sampling rate - the time interval between two consecutive samples, or bin - according to the needs and objectives of the undergoing experiment. In this age of fast computers and large hard drives, the trend is to choose a very high frequency of sampling and disregard the storage size; however, sample size does matter and it is a key factor for further data analysis. The sampling rate will define the shortest cycle that can be measured. According to the Nyquist theorem, the sample rate must be at least twice the higher frequency (i.e., $1 / \tau$ ) that it is aimed to study (Levine, Funes, Dowse, \& Hall, 2002). For example, if we aim only to find the circadian frequency (24-h), we could just sample every 12 hours. However, in this case we cannot compute a phase or an accurate period. On the other hand, if the sampling rate were once per hour, then it would be possible to evaluate periodicities down to two hours (but not shorter), since a minimum of two points is required to describe a cycle. For this reason, the precision of the experimental approach is limited by the sampling procedure. However, a very high sampling rate would implicate low sample size defining sample size as the length of the integrated time of the measure. In this case, when a very low sample size is used, the time for data processing and visualization greatly increases. According to the experimental output (e.g., locomotor activity, gene or protein oscillation, etc.), a compromise is reached between accuracy and speed. The usual sample size for circadian analysis of locomotor activity data varies between 5 to 30 minutes. This sample size will allow an accurate determination of circadian period and phase, as well as the study of the transient behavior of these parameters (including period and phase shift).

Sometimes there are some elements in the signal that may interfere with the calculation of circadian parameters. In this case, the raw data often need to be filtered for further study; this processing of the raw signal before the analysis is known as signal conditioning. Two of these interfering elements will be addressed in this section: the presence of a shifting temporal baseline (i.e., linear trend) and the presence of a high frequency noise.

In the first case (the presence of a shifting temporal baseline), the two types of linear effects that can alter the subsequent data analyses involve: 
(1) a monotonically decrease or increase in the signal at a constant rate, thus producing a linear trend in the data, and (2) the rhythmic component of a signal can be obscured by high baseline activity. The first type of linear effect may have linear as well as non-linear components. This trend can be caused, for instance, by the natural aging of invertebrate models and it is common in their locomotor activity recordings. The second type of linear effect, a high baseline, could occur in many cases (e.g., data from imaging experiments). Even though the rhythm is quite strong in the circadian range, it cannot be computed before the conditioning. Removing this kind of trend is quite common and is highly recommended. It can be simply done by removing a regression line, to obtain a signal with slope and mean of 0 . When the trend is non-linear, this detrend is a bit more complex; the most common approach is to use a moving-average window of fixed length to obtain the non-linear trend over time. Once we have obtained the trend, we can remove it with an amplitude correction by dividing the original signal by the trend, or by subtracting the signal. Thus, we keep the natural baseline of the signal, but we remove the non-linear trend. Detailed examples for detrending and normalizing a signal for circadian analysis can be found in Levine et al., 2002.

In the second case (the presence of high frequency noise), the approach is to use digital filters. These filters diminish the spectral contribution of a certain wavelength to the signal while keeping the others intact. The filters that reduce the high frequency components are called low-pass or smooth. We will mention a simple filter, moving-average smoothing, but there are other types of filters more complex, with different advantages and disadvantages. A filter can be defined as a function that transforms a time series into another. The moving-average filter is one of the most simple and used in the circadian field. It is used to analyze a set of data by creating a series of averages of different subsets of the full data set. Specifically, this filter averages a fixed number of points - it is better to have an odd number of points - in order to find the mean value of the initial subset of data. Then the subset is modified by moving one point forward in time, and the process is repeated until we reach the end of the data series. The resulting plot line connecting all the averages is the moving average: a set of numbers, each of which is the average of the corresponding subset of a larger set of data. In this case we give each point the same weight, but sometimes it is recommended weighting differently each point mainly with a Gaussian distribution (Levine et al., 2002) to give the central point of the window a higher weight. An example of moving average can be found in the online Appendix section (smoothma.m file; see book's GitHub repository). 


\subsection{Actograms}

As previously shown in Figures 1.2 and 1.3, actograms are a useful tool to represent circadian locomotor activity rhythms in several species. The actogram is usually performed as a 'double plot' graph, where two cycles are represented in the same horizontal line. Specifically, double-plotted actograms show the second cycle on the right side of each line, as well as at the start of the following horizontal line, and so on. If the actogram is usually set at a fixed period of $24-\mathrm{h}$ (for a solar day) in the $\mathrm{x}$-axis, then the double plot representation will have 48-h duration (Figure 1.5, left panel). The cycle is divided into a time fraction with main activity episodes (called $a l p h a$ ) and other with major rest episodes (called rho). Sometimes, scattered activity can be observed in the rho time because the animal interrupts its sleep for a short time (Jud et al., 2005). Doubleplotting is especially useful to visualize non-24-h rhythms or very long series.

One of the reasons that the actogram became a key representation in Chronobiology is that it allows a fast and reliable visual analysis. With a simple observation we can realize the changes of period and phase. The actogram was first introduced by Colin Pittendrigh at the beginning of the 1960 (Pittendrigh, 1965), well before any computational method for circadian data analysis was established. By that time, data were plot and measured by hand. Currently, there are many computational tools (e.g., a circadian plugin for Imagej and the software El Temps) that still maintain the concept of visual analysis ('paper and ruler' analysis, in which the actogram is printed and analyzed with common measuring instruments).

The main downfall of this approach is the variability of the observation between users and the lack of rigorous quantitative analysis. Nevertheless, this kind of analysis is still heavily used. A quick example of this visual inspection in the actogram can be seen in Figure 1.6, where locomotor records with different periods, as well as phase shifts, can be easily identified.

An example of a double-plotted actogram containing real data can be found in the online Appendix, by running the function Actogram (see the files SCRIPT.m and ScriptFilter.m for further details; see book's GitHub repository).

\subsection{Waveform}

Periodic data of known period could be divided into sections, with the length of these sections matching the underlying period. Overlaying these sections (cycles) will produce a clear waveform, with peak and trough (Figure 1.5, right panel). In other words, a waveform can be thought as an average of all the lines of the actogram. The length of the waveform is given by the length of the cycle, which can be empirically fixed (e.g., the length of a light/dark cycle) or previously calculated by any method. Even though it can be used in many 
cases, this technique is more common with evenly sampled series (uniform sampling) containing a good number of complete cycles (10 or higher). The observation and analysis of the average wave yields important characteristics of the rhythm, such as the duration of the activity time (alpha) and the rest time $(r h o)$. To define these different states of activity we need to find the beginning of the main activity episode, called activity onset, and the end or activity offset, which in many cases is a quite easy task. However, when these points are more difficult to find, as for instance due to fragmentation of activity episodes, is better to set an objective criterion. One approach is to find the median or the average of activity within the waveform cycle. Thus, when the activity goes over this threshold for a certain amount of time, we can define a statistical criterion for the activity onset. Similarly, when the activity falls under this threshold for the same amount of time, we can define the activity offset. The time difference between activity offset and onset is used to calculate alpha (see Figure 1.5).
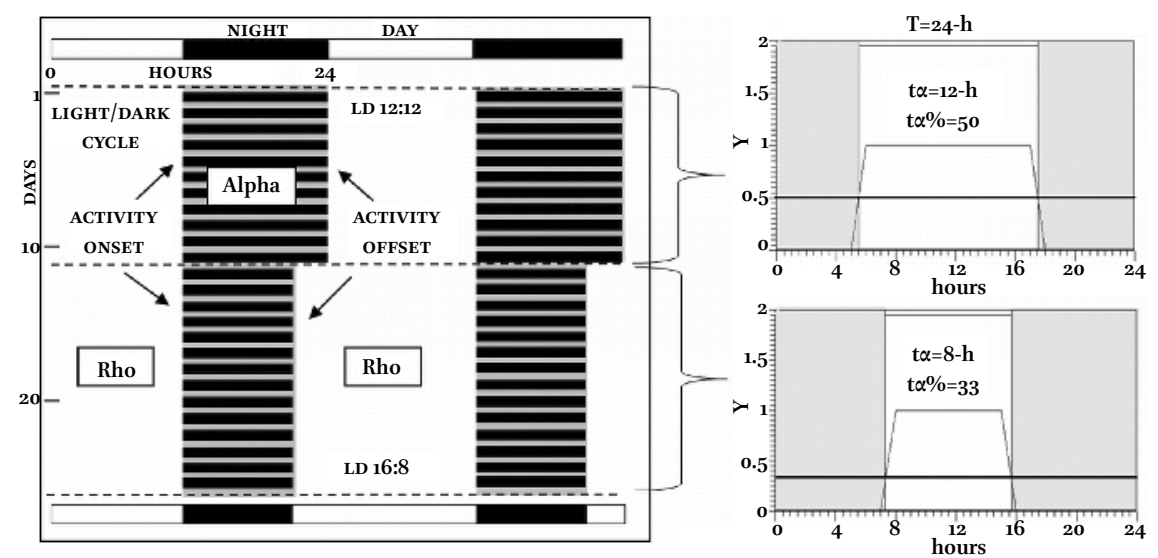

FIGURE 1.5 Double-plotted actograms and their respective waveforms. To better visualize behavioral rhythms, actograms (left panel) are often double-plotted by aligning two consecutive days horizontally (e.g., day 1 left and day 2 right). In this example, two different light/dark $(L D)$ conditions with a period of 24-h are shown: a LD 12-12 cycle (i.e., 12-h of light and 12-h of darkness), and a LD 16-8 cycle (i.e., 16- $h$ of light and 8-h of darkness). Activity onset, activity offset, alpha and rho are indicated in the actogram. The resulting waveforms (right panel) for these two LD conditions display the same period (24-h). In each waveform, the length of the activity portion (alpha, white background) is measured between the activity onset and the activity offset, being $t \alpha=12-h$ for the upper waveform and $t \alpha=8$ - $h$ for the bottom waveform. The percent of this length, according to the total length of the cycle (alpha percentage), is $50 \%$ and $33 \%$, respectively. The length of the rest time rho $(t \rho)$ and its percentage $(t \rho \%)$ can be obtain as the complement of $t \alpha$ and $t \alpha \%$ respectively. 
Once the limits of alpha and rho phases are defined, their length (t $\alpha$ and $\mathrm{t} \rho$ ) is easily measured by taking into account that $\mathrm{T}=\mathrm{t} \alpha+\mathrm{t} \rho$. The most usual way to represent these times is relative to the length of $\mathrm{T}$ (for alpha $\mathrm{t} \alpha \%=$ 100 t $\alpha / \mathrm{T}$ ). For example, for $\mathrm{T}=24-\mathrm{h}$ and $\mathrm{t} \alpha=12-\mathrm{h}$, then t $\alpha \%=5^{0}$, meaning that half the total period corresponds to the activity phase. Along with the length of alpha and rho phases, it can be also calculated the area under the curve for both time lapses ( $\mathrm{A} \alpha$ and $\mathrm{A} \rho$ ) and to express them as percentages of the total area (A $\alpha \%$ and $A \rho \%)$. Finally, with both the length and amount of activity in both states, it can be computed the degree of agglomeration $(\mathrm{A} \alpha \% / \mathrm{t} \alpha \%)$. When this degree is near 1 , the distribution of activity is uniform (i.e., we have a weak variation in the rhythm), while when this degree is higher than 1, most of the activity is concentrated in the alpha phase (Madrid \& de Lama, 2006).

\subsection{Periodogram}

A key step in the analysis of circadian data is to accurately calculate the underlying period. There are several methods for determining period, which range from graphic approaches, such as the actogram - mentioned in Section 6.3to more mathematically complex approaches, such as Fourier-transform and non-Fourier-transform based algorithms (Zielinski, Moore, Troup, Halliday, \& Millar, 2014). The latter methods allow a level of significance for period calculation, as well as an unbiased estimation.

A periodogram is a function relating periodic components of a time series to their spectral power. Many algorithms for period estimation had been described in the literature, all of them with a varying degree of complexity and different assumptions. Nevertheless the calculation method, all periodograms possess the same graphic representation, which makes them quite easy to understand (Figure 1.6). Several authors (Refinetti, 1993; Zielinski et al., 2014) compare the different methods for periodogram analysis (e.g., Fourier, Enright, Lomb-Scargle, etc.) and describe the best option for different cases. Each method has its own strength and weakness. In this section, we will mention two widely used methods: Lomb-Scargle (LS, very useful in short series and non-uniform sampling) and the Chi-square method, also known as Sokolove-Bushell (sB).

The most popular and conceptually simpler method is the Chi-square periodogram - its name corresponds to the statistical distribution that it uses. This method consists in splitting the data in sections of a fixed length. When this fixed length matches the period of the series, the overlay of all sections will give a clear waveform (with a strong variation) as the repeating patterns of the series coincide. However, if the length of sections does not coincide, then the 
(A)
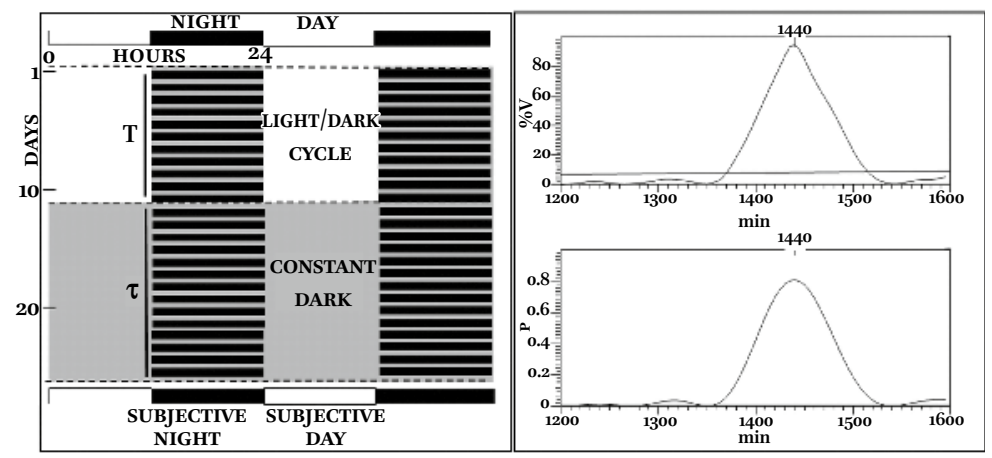

(B)

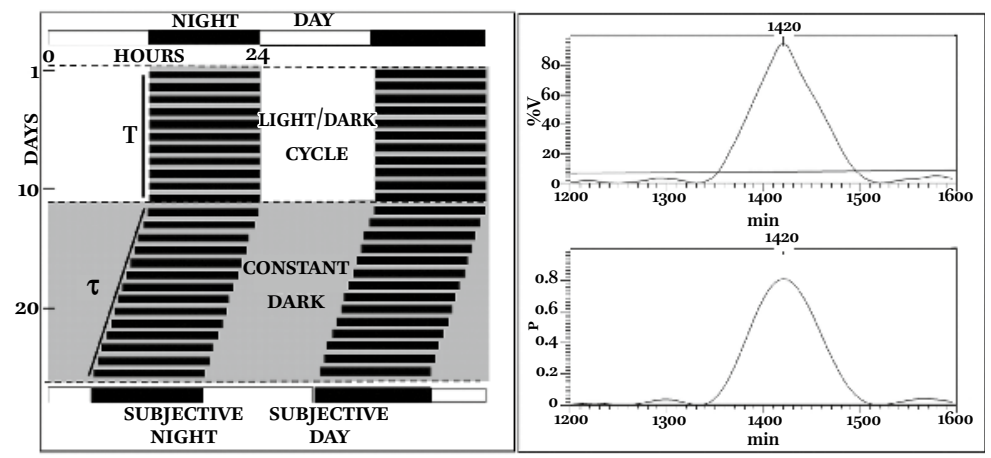

(C)

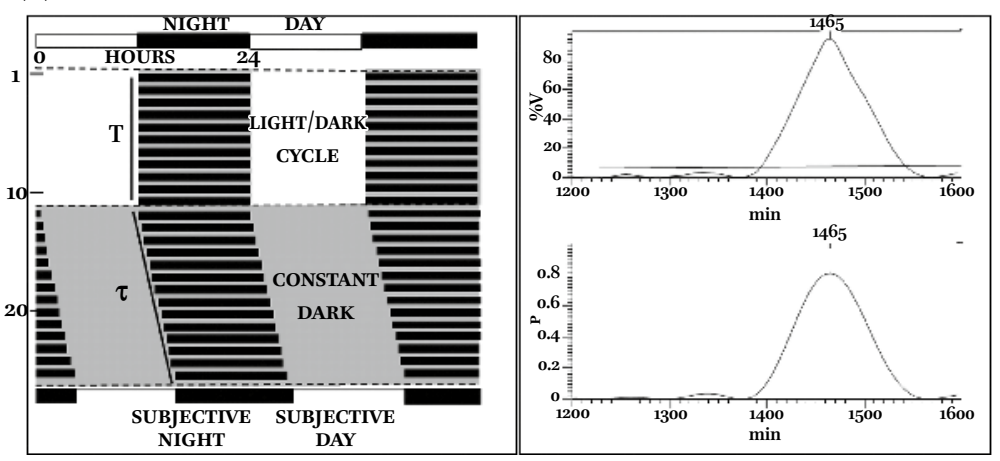

FIGURE 1.6 Double-plotted actograms and their respective periodograms. $(A) T=\tau$, with a period of 1440 minutes $(24-h)$. (B) When $\tau<T$, the activity seems to drift left. In this example, the endogenous period is $\tau=1420$ minutes. $(C)$ When $T<\tau$, the activity drifts to the right. In this example, the endogenous period is $\tau=1465 \mathrm{~min}$. In all cases, the peridogram represents the constant dark condition. Upper panel: Sokolov-Bushel $(s в)$ periodogram. Lower panel: Lomb-Scargle (LS) periodogram. It is also possible to have an idea of the resulting period by the actogram's visual inspection. By using the proper tool, the endogenous period can be calculated as the slope of the change in $\tau$ ('paper and ruler' method). 
peaks and troughs will not match, and overlaying the sections together will result in a small-amplitude signal with a non-significant variation. To analyze data with unknown period, the algorithm evaluates a wide range of length for different periods. When the maximum correlation is found - and it is statistically significant - we have found the main period of the series that gave the highest amplitude of the waveform. One of the best features of this method is its independence from the waveform of the rhythmic signal, because it only requires a repeating pattern, while other methods, such as Fourier or LS, assume a sinusoidal waveform. The main downfall of the sв method is that the step size between periods that can be tested is limited by the sample size and the duration of the series. This method is widely used in long time series (more than 10 cycles) with a high sampling rate.

Another general approach is based in the curve-fitting criterion. This method assumes an a-priori periodic signal (mainly sinusoidal) and optimizes the signal parameters to best fit the data. In this case, we plot the correlation coefficient after the fit with different periods. This approach allows using shorter series and even non-uniform sampling. The Fourier method is the most basic approach of this set of algorithms. As we use a sinusoidal signal to fit, the method is especially sensitive to this waveform; however, it is not as good to fit other types of rhythmic signals. The Lomb-Scargle periodogram consists of a spectral analysis (a modification of the Fourier periodogram). This method creates a spectrum representing the significance of each frequency with a normalized power, and is usually the best choice in short series and non-uniform sampling. This method combines both cosine and sine fit to find periodicity regardless of the data phase. Another interesting feature is that it reduces the subsequent sub-harmonics of the main period (if the period is $\mathrm{T}$, the peaks in $2 \mathrm{~T}, 3 \mathrm{~T}$, etc.) and, therefore, the periodogram analysis and representation are 'clean'.

There are many other methods to calculate the underlying period, such as MESA (Maximum Entropy Spectral Analysis) or FFT-NLls (Fast Fourier Transform Non-linear Least Square algorithm). MESA is very useful because it can resolve multiple periods that are relatively close (Dowse, 2013). It is beyond the objective of the present chapter to describe all of them, but it is important to recognize which method is the best choice according to the specific experimental data. A comparison of the most used algorithms for determining period can be found in Zielinski et al. (2014).

An example containing the periodogram calculation of real data can be found in the online Appendix, by running the functions chisquare (Chisquare or Sokolov-Bushel periodogram) and lsssigx (a simplify application of the fastlomb.m that gives the complete spectrum of the Lomb-Scargle 
periodogram). See the files SCRIPT.m and ScriptFilter.m for further details (see book's GitHub repository).

\subsection{Cosinor}

As it was already shown in Figure 1.4, a simple rhythm can be described as a sinusoidal wave. By taking into account all the rhythm parameters, the signal can be mathematically expressed as:

$$
y=M+A \cos (\omega t-\varphi)
$$

where $\mathrm{M}$ is the mean of the adjusted data (Midline Estimating Statistic Of Rhythm, MESOR), $\varphi$ is the acrophase, $A$ is the amplitude (difference between $\varphi$ and $M)$ and $t$ corresponds to time. Finally, $\omega=2 \pi / T$ is the angular frequency, being $\mathrm{T}$ the rhythm period. The mathematical procedure that fits the best curve - best fit according to the less-square method - is known as Cosinor. The expression of $y$ leads to a nonlinear optimization to find the values of $\mathrm{M}, \mathrm{A}$ and $\varphi$. The equation (1), by using the Euler's equation through the formula $\cos (\alpha-\beta)=\cos (\alpha) \cos (\beta)+\sin (\alpha) \sin (\beta)$, is transformed to:

$$
y=M+\alpha \cos (\omega t)+b \sin (\omega t)
$$

where $a[\mathrm{~A} \cos (\varphi)]$ and $b[\mathrm{~A} \sin (\varphi)]$ are known as the Euler coefficients, and are used to calculate the values of $A$ and $\varphi$ in the original equation (1) as follows:

$$
A=\sqrt{a^{2}+b^{2}} ; \varphi=\tan ^{-1}(b / a)
$$

If the signal was uniform sampled, the calculation is even simpler:

$$
M=\frac{1}{n} \sum_{i=1}^{n} y ; a=\frac{2}{n} \sum_{i=1}^{n} y_{i} \cos \left(\omega t_{i}\right) ; b=\frac{2}{n} \sum_{i=1}^{n} y_{i} \sin \left(\omega t_{i}\right)
$$

The Cosinor method is mainly used to analyze a rhythmic time series with a known period (T), which can be obtained from the periodogram analysis (Cornelissen, 2014; Diez-Noguera, 2013). The Cosinor analysis will allow to obtain the phase $(\varphi)$ and the amplitude (A) of the rhythm. A polar graph is usually performed to plot the results (see Figure 1.7 and online Appendix). In this graph, a circle represents the period $\mathrm{T}$, with a vector that origins in the center of the circle. This vector has a size that depends on the amplitude (A) and its direction depends on the time of the acrophase $(\varphi)$. Additionally, it is 
(A)
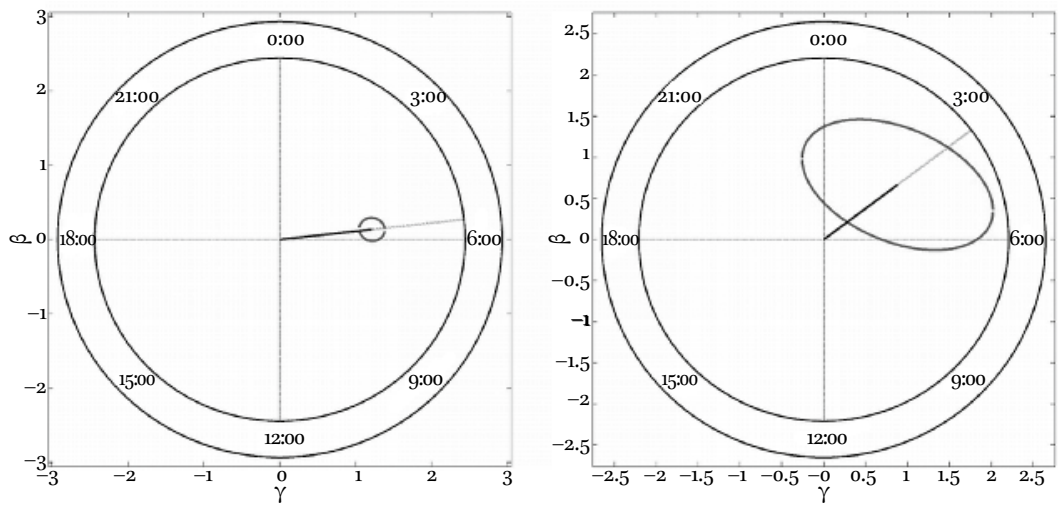

(B)
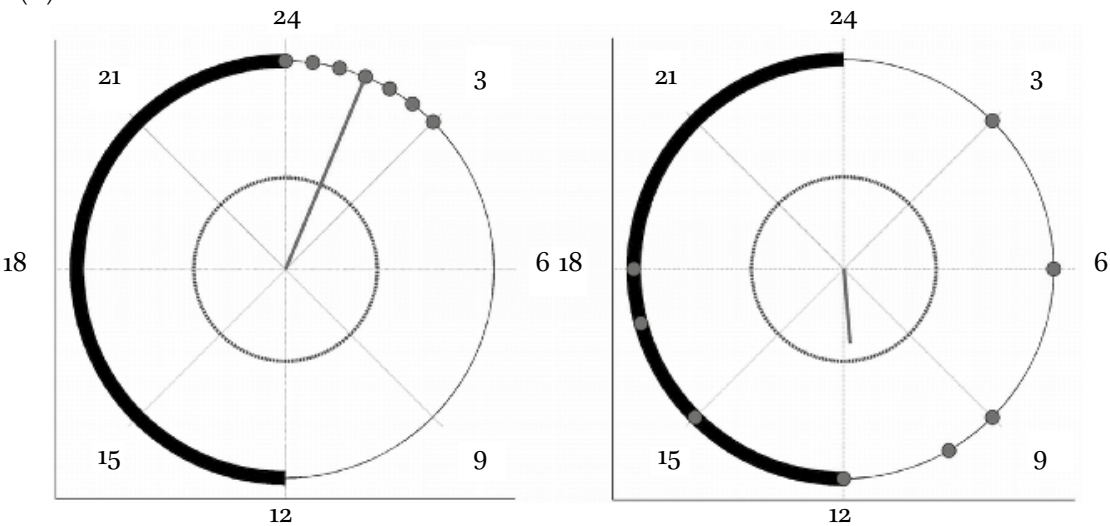

FIGURE 1.7 Graphical representation of Cosinor and Rayleigh in a polar plot. $(A)$ Cosinor analysis of two data sets. This is illustrated by the elliptical 95\% confidence interval (cI) for the amplitude-acrophase pair. The $95 \%$ cI is located at the endpoint of the vector. In the left panel, the phase can be found and is significant as it pass the null amplitude hypothesis (Mesor $=0.019 ;$ Amplitude $=1.22$; Acrophase $=-1.46$ radians $=$ 5.57 hours; $p<0.05)$. In the right panel, the data does not pass this test $($ Mesor $=0.12$; Amplitude $=1.10$; Acrophase $=-0.92$ radians $=3.51$ hours; $p>0.05) .(B)$ Rayleigh test of two data sets. In the left panel there is a significant unimodal aggrupation $(p=2 e-4)$, while and in the right panel there is a non-significant data set $(p=0.38)$. The significance can be clearly observed as the resultant vector goes beyond the significance circle in the center of the left panel graph, while it does not go beyond the circle in the right panel.

usually represented in the same graph the confidence interval (CI) for each parameter, forming an ellipse around the further point of the vector. When the ellipse includes the vector's originating point, then the amplitude (and thus the rhythm) is statistically non-significant (i.e., A is non-different from zero). This is caused when the fitted curve represents less than $60 \%$ of the total 
variation of the signal ( $40 \%$ residual variation of the less-squared fitting). In a similar way, when more than one signal is represented in the same graph, a superposition of the CI indicates that the two signals do not have a significant difference.

The Cosinor method is widely used to find the amplitude (as criterion for significant rhythmicity), but more importantly, the acrophase of the time series. However, as many other curve-fitting methods, is strongly dependent on the signal's waveform, and responds much better (both in accuracy and significance) with smooth sinusoidal data than signals with abrupt changes or nonsymmetrical shapes (Cornelissen, 2014).

The online Appendix includes examples of Cosinor analysis of locomotor activity series. To analyze this kind of data, first select the time period and then run the function cosinor (see the files SCRIPT.m and ScriptFilter.m for further details in book's GitHub repository).

\subsection{Circulars Statistics and Rayleigh}

Circular statistics refers to the development of statistical techniques for the use with data represented on an angular scale (Berens, 2009). Due to their circular nature, many circadian data can be analyzed with circular statistics. The phase of time series, when we study a rhythmic signal, is an example of circular data. In this case, there is no designated zero and, in contrast to a linear scale, high/low values are arbitrary. This idea of 'circular time' is especially strong in the case of analyzing repeating patterns with a fixed time range (i.e., a cycle of period $\mathrm{T}$ and how this pattern changes). For this reason, the rhythmic signal is naturally represented as a circular graph (as shown in the Cosinor's section). The rhythm's phase can be studied as a fraction of the complete cycle (in time, angle or radians).

There are many other tests for circular statistics as an alternative for Rayleigh, such as the Omnibus test (Zar, 1999). This test works well with unimodal, bimodal and multimodal data, but its use is more selective and is not as extensive as others (Berens, 2009).

In case that there is a set of signals (e.g., many individuals in the same experiment), each one will have its own phase. We can study phase similarities by representing all the phases in the same circular graph and test them for a significant clustering by means of the Rayleigh test (Figure 1.7). This test asks how large the vectorial sum of the individual vectors must be to indicate a non-uniform distribution, according to the Fisher circular distribution (Fisher, 1995). The Rayleigh test is especially strong for detecting a unimodal deviation from uniformity (Berens, 2009). The test estimates the module (r) of the sum vector of the unit vectors corresponding to the phases of each individual. The higher the value of $r$, the greater degree of phase homogeneity. The $p$-value of 
the data can be computed by obtaining the critical value from the distribution. By setting the significance threshold in $\mathrm{p}=0.05$ the critical value of the vector can be obtained beforehand. In this way, we can approximate the critical value $=\mathrm{r} \approx 1.673^{2268} /(1-\mathrm{n}) 0.492018$, where $\mathrm{n}$ is the number of cases used (Diez-Noguera, 2013).

Examples of circular statistics can be found in the online Appendix section (Rayleigh.m file; see book's GitHub repository).

\section{Conclusions}

The circadian system is ubiquitous, being essential for most living organisms' physiology. The interaction of several circadian clock genes and proteins generates oscillations in expression of output target genes which temporally regulate numerous molecular and cellular processes. A precise regulation of circadian rhythms is crucial to keep track with the environment, and perturbations in the circadian system are linked to numerous molecular dysfunctions and may result in severe pathologies.

Hence, a comprehensive knowledge regarding the mechanistic of the circadian system is necessary to develop new procedures to investigate pathologies associated with a deregulated clock.

In the online appendix section (see book's GitHub repository), we incorporate important tools for circadian data analysis, including codes and sample data. We include the codes for LS, SB, Cosinor, and Rayleigh analysis as well as a code to plot actograms. There are many other software - some of them freeware - that possess a wider range of circadian tools. We include links to some software too.

\section{References}

Agostino, P.V., \& R.K. Cheng (2016). Contributions of dopaminergic signaling to timing accuracy and precision. Current Opinion in Behavioral Sciences, 8, 153-160.

Agostino, P.V., M. do Nascimento, I.L. Bussi, M.C. Eguía, \& D.A. Golombek (2011). Circadian modulation of interval timing in mice. Brain Research, 1370, 154-163.

Agostino, P.V., S.A. Plano, \& D.A. Golombek (2007). Sildenafil accelerates reentrainment of circadian rhythms after advancing light schedules. Proceedings of the National Academy of Science U S A, 104, 9834-9839.

Albrecht, U. (2012). Timing to perfection: The biology of central and peripheral circadian clocks. Neuron, 74, 246-260. 
Aschoff, J. (1985). On the perception of time during prolonged temporal isolation. Human Neurobiology, 4, 41-52.

Aschoff, J., S. Daan, \& K.I. Honma (1982). Zeitgeber, entrainment, and masking: Some unsettled questions. In Aschoff, J., S. Daan, G.A. Gross, Vertebrate Circadian System (Structure and Physiology). Berlin: Springer-Verlag.

Bell-Pedersen, D., V.M. Cassone, D.J. Earnest, S.S. Golden, P.E. Hardin, T.L. Thomas, \& M.J. Zoran (2005). Circadian rhythms from multiple oscillators: Lessons from diverse organisms. Nature Reviews Genetics, 6(7), 544-556.

Berens, P. (2009). CircStat: A MAT LAB Toolbox for Circular Statistics. Journal of Statistical Software, 31(10), 1-21.

Bonmati-Carrion, M.A., B. Middleton, V. Revell, D.J. Skene, M.A. Rol, \& J.A. Madrid(2014). Circadian phase assessment by ambulatory monitoring in humans: Correlation with dim light melatonin onset. Chronobiology International, 31(1), 37-51.

Bordyugov, G., U. Abraham, A. Granada, P. Rose, K. Imkeller, A. Kramer, \& H. Herzel (2015). Tuning the phase of circadian entrainment. Journal of The Royal Society Interface, 12(108), 20150282.

Buhr, E.D., \& J.S. Takahashi (2013). Molecular components of the mammalian circadian clock. Handbook of Experimental Pharmacology, 217, 3-27.

Buhusi, C.V., \& W.H. Meck (2005). What makes us tick? Functional and neural mechanism of interval timing. Nature Reviews Neuroscience, 6(10), 755-765.

Bussi, I.L., G. Levin, D.A. Golombek, \& P.V. Agostino (2015). Melatonin modulates interval timing in rats: Effect of pinealectomy. International Journal of Comparative Psychology, 28(1).

Bussi, I.L., G. Levín, D.A. Golombek, \& P.V. Agostino (2014). Involvement of dopamine signaling in the circadian modulation of interval timing. European Journal of Neuroscience, 4o(1), 2299-2310.

Campuzano, A., J. Vilaplana, T. Cambras, \& A. Diez-Noguera (1998). Dissociation of the rat motor activity rhythm under T cycles shorter than 24 hours. Physiology \& Behavior, 63(2), 171-176.

Casiraghi, L.P., G.A. Oda, J.J. Chiesa, W.O. Friesen, \& D.A. Golombek (2012). Forced desynchronization of activity rhythms in a model of chronic jet lag in mice. Journal of Biological Rhythms, 27(1), 59-69.

Castanon-Cervantes, O., M. Wu, J.C. Ehlen, K. Paul, K.L. Gamble, R.L. Johnson, R.C. Besing, M. Menaker, A.T. Gewirtz, \& A.J. Davidson (2010). Dysregulation of inflammatory responses by chronic circadian disruption. The Journal of Immunology, 185(10), 5796-5805.

Chandrashekaran, M.K., G. Marimuthu, R. Subbaraj, P. Kumarasamy, M.S. Ramkumar, \& K. Sripathi (1991). Direct correlation between the circadian sleep-wakefulness rhythm and time estimation in humans under social and temporal isolation.Journal of Biosciences. 16, 97-101. 
Cho, K. (2001). Chronic 'jet lag' produces temporal lobe atrophy and spatial cognitive deficits. Nature Neuroscience, 4, 567-568.

Cho, K., A. Ennaceur, J.C. Cole, \& C.K. Suh (2000). Chronic jet lag produces cognitive deficits. The Journal of Neuroscience, 20(6), 1-5.

Clayton, M., R. Sager, \& U. Will (2005). In time with the music: The concept of entrainment and its significance for ethnomusicology. European meetings in ethnomusicology, 11, 1-82.

Cornelissen, G. (2014). Cosinor-based rhythmometry. Theoretical Biology and Medical Modelling, $11(1), 1$.

de la Iglesia, H.O., T. Cambras, W.J. Schwartz, \& A. Diez-Noguera (2004). Forced desynchronization of dual circadian oscillators within the rat suprachiasmatic nucleus. Current Biology, 14(9), 796-80o.

Devan, B.D., E.H. Goad, H.L. Petri, E.A. Antoniadis, N.S. Hong, C.H. Ko, L. Leblanc, S.S. Lebovic, Q. Lo, M.R. Ralph, \& R.J. McDonald (2001). Circadian phase-shifted rats show normal acquisition but impaired long-term retention of place information in the water task. Neurobiology of learning and memory, 75(1), 51-62.

Díez-Noguera, A. (2013). Methods for serial analysis of long time series in the study of biological rhythms. Journal of circadian rhythms, $11(1), 7$.

Dowse, H.B. (2013). Maximum entropy spectral analysis for circadian rhythms: Theory, history and practice. Journal of circadian rhythms, n(1), 6.

Dunlap, J.C., J.J. Loros, \& P.J. DeCoursey (2004). Chronobiology. Biological timekeeping. Sunderland, MA: Sinauer Associates, Inc.

Eckel-Mahan, K.L., \& D.R. Storm (2009). Circadian rhythms and memory: not so simple as cogs and gears. Емво Reports, $10,584-591$.

Fisher, N.I. (1995). Statistical analysis of circular data. Cambridge, uk: Cambridge University Press.

Folkard, S. (1996). Effects on performance efficiency. In Coquhoun, W.P., G. Costa, S. Folkard, and P. Knauth (Eds.), Shiftwork: Problems and Solution (pp. 65-87). Frankfurt: Peter Lang.

Fonken, L.K., M.S. Finy, J.C. Walton, Z.M. Weil, J.L. Workman, J. Ross, \& R.J. Nelson (2009). Influence of light at night on murine anxiety- and depressive-like responses. Behavioural Brain Research, 205(2), 349-354.

Fonken, L.K., J.L. Workman, J.C. Walton, Z.M. Weil, J.S. Morris, A. Haim, \& R.J. Nelson (2010). Light at night increases body mass by shifting the time of food intake. Proceedings of the National Academy of Science USA, 107(43), 18664-18669.

Gerstner, J.R., \& J.C. Yin (2010). Circadian rhythms and memory formation. Nature Reviews Neuroscience, $11,577-588$.

Gekakis, N., D. Staknis, H.B. Nguyen, F.C. Davis, L.D. Wilsbacher, D.P. King, J.S. Takahashi, \& C.J. Weitz (1998). Role of the CLOCK protein in the mammalian circadian mechanism. Science, 280, 1564-1569. 
Gibson, E.M., C. Wang, S. Tjho, N. Khattar, \& L.J. Kriegsfeld (2010). Experimental 'jet lag' inhibits adult neurogenesis and produces long-term cognitive deficits in female hamsters. PLoS One 5, e15267.

Golombek, D.A., \& R.E. Rosenstein (2010). Physiology of circadian entrainment. Physiological reviews, 9o, 1063-1102.

Golombek, D.A., P.V. Agostino, S.A. Plano, \& G.A. Ferreyra (2004). Signaling in the mammalian circadian clock: The NO/cGMP pathway. Neurochemistry International, 45, 929-936.

Golombek, D.A., I.L. Bussi, \& P.V. Agostino (2014). Minutes, days and years: Molecular interactions among different scales of biological timing. Philosophical Transactions of the Royal Society of London B: Biological Sciences, 369(1637), 20120465.

Golombek, D.A., L.P. Casiraghi, P.V. Agostino, N. Paladino, J.M. Duhart, S.A. Plano, \& J.J. Chiesa (2013). The times they're a-changing: Effects of circadian desynchronization on physiology and disease. Journal of Physiology-Paris, 107(4), 310-322.

Golombek, D.A., G.A. Ferreyra, P.V. Agostino, A.D. Murad, M.F. Rubio, G.A. Pizzio, M.E. Katz, L. Marpegan, \& T.A. Bekinschtein (2003). From light to genes: Moving the hands of the circadian clock. Frontiers of Bioscience, 8, s285-293.

Gooley, J.J. (2008). Treatment of circadian rhythm sleep disorders with light. Annual Academy of Medice Singapore, 37, 669-676.

Goya, M.E., A. Romanowski, C.S. Caldart, C.Y. Bénard, \& D.A. Golombek (2016). Circadian rhythms identified in Caenorhabditis elegans by in vivo long-term monitoring of a bioluminescent reporter. Proceedings of the National Academy of Science USA, $113(48)$, E7837-E7845.

Gritton, H.J., A. Kantorowski, M. Sarter, \& T.M. Lee (2012). Bidirectional interactions between circadian entrainment and cognitive performance. Learning \& Memory, 19(3), 126-141.

Guillaumond, F., H. Dardente, V. Giguère, \& N. Cermakian (2005). Differential control of Bmalı circadian transcription by REV-ERB and ROR nuclear receptors. Journal of Biological Rhythms, 20, 391-403.

Hampp, G., J.A. Ripperger, T. Houben, I. Schmutz, C. Blex, S. Perreau-Lenz, I. Brunk, R. Spanagel, G. Ahnert-Hilger, J.H. Meijer, \& U. Albrecht (2008). Regulation of monoamine oxidase A by circadian-clock components implies clock influence on mood. Current Biology, 18(9), 678-683.

Johnson, C.H., J.A. Elliott, \& R. Foster (2003). Entrainment of circadian programs. Chronobiology International, 20, 741-774.

Jones, M.R. (1976). Time, our lost dimension: Toward a new theory of perception, attention, and memory. Psychological Review, 83(5), 323.

Jones, C.R., S.S. Campbell, S.E. Zone, F. Cooper, A. DeSano, P.J. Murphy, B. Jones, L. Czajkowski, \& L.J. Ptč (1999). Familial advanced sleep-phase syndrome: A shortperiod circadian rhythm variant in humans. Nature Medicine, 5(9), 1062-1065. 
Jud, C., I. Schmutz, G. Hampp, H. Oster, \& U. Albrecht (2005). A guideline for analyzing circadian wheel-running behavior in rodents under different lighting conditions. Biology Proceeding Online, 7, 101-116.

Karatsoreos, I.N., S. Bhagat, E.B. Bloss, J.H. Morrison, \& B.S. McEwen (2011). Disruption of circadian clocks has ramifications for metabolism, brain, and behavior. Proceedings of the National Academy of Science USA, 108, 1657-1662.

Klerman, E.B., H.B. Gershengorn, J.F. Duffy, \& R.E. Kronauer (2002). Comparisons of the variability of three markers of the human circadian pacemaker. Journal of Biological Rhythms, $17(2)$, 181-193.

Kuriyama, K., M. Uchiyama, H. Suzuki, H. Tagaya, A. Ozaki, S. Aritake, K. Shibui, T. Xin, L. Lan, Y. Kamei, \& K. Takahashi (2005). Diurnal fluctuation of time perception under 30-h sustained wakefulness. Neuroscience Research, 53, 123-128.

Kyriacou, C.P., \& J. Hall (1980). Circadian rhythm mutations in Drosophila melanogaster affect short-term fluctuations in the male's courtship song. Proceedings of the National Academy of Science US A, 77, 6729-6733.

Kyriacou, C.P., \& M.H. Hastings (2010). Circadian clocks: Genes, sleep, and cognition. Trends in Cognitive Sciences, 14, 259-267.

LeSauter, J., \& R. Silver (1998). Output signals of the scN. Chronobiology International, 15(5), 535-550.

Levine, J.D., P. Funes, H.B. Dowse, \& J.C. Hall (2002). Signal analysis of behavioral and molecular cycles. BMC Neuroscience, $3(1), 1$.

Lewy, A.J., J. Emens, A. Jackman, \& K. Yuhas (2006). Circadian uses of melatonin in humans. Chronobiology International, 23(1-2), 403-412.

Loh, D.H., J. Navarro, A. Hagopian, L.M. Wang, T. Deboer, \& C.S. Colwell (2010). Rapid changes in the light/dark cycle disrupt memory of conditioned fear in mice. PLoS One, 5, e12546.

Lowrey, P.L., \& J.S. Takahashi (200o). Genetics of the mammalian circadian system: Photic entrainment, circadian pacemaker mechanisms, and posttranslational regulation. Annual Review of Genetics, 34(1), 533-562.

Lustig, C., \& W.H. Meck (2001). Paying attention to time as one gets older. Psychological Science, $12(6), 478-484$.

Madrid, J.A., \& A.R. de Lama (2006). Cronobiología básica y clínica. Editec@ red. Madrid, España.

Madrid, J.A., P. Lax, J. Vilaplana, T. Cambras, \& A. Diez-Noguera (1992). Presence of two differentiated circadian components in the eating and motor behaviour in the young rat. Journal of Interdisciplinary Cycle Research, 23, 211-212.

Mahoney, M.M. (2010). Shift work, jet lag, and female reproduction. International Journal of Endocrinology, 2010, 813764. 
Meck, W.H. (1991). Modality-specific circadian rhythmicities influence mechanisms of attention and memory for interval timing. Learning and Motivation, 22(1), 153-179.

Meng, Q.J., E.S. Maywood, D.A. Bechtold, W.Q. Lu, J. Li, J.E. Gibbs, S.M. Dupré, J.E. Chesham, F. Rajamohan, J. Knafels, B. Sneed, L.E. Zawadzke, J.F. Ohren, K.M. Walton, T.T. Wager, M.H. Hastings, \& A.S. Loudon (2010). Entrainment of disrupted circadian behavior through inhibition of casein kinase 1 (CK1) enzymes. Proceedings of the National Academy of Sciences USA, $107(34), 15240-15245$.

Morin, L. (2013). Neuroanatomy of the extended circadian rhythm system. Experimental Neurology, 243, 4-20.

Morin, L.P., \& C.N. Allen (2006). The circadian visual system. Brain Research Reviews, $51(1), 1-60$.

Moriya, T., Y. Yoshinobu, Y. Kouzu, A. Katoh, H. Gomi, M. Ikeda, T. Yoshioka, S. Itohara, \& S. Shibata (200o). Involvement of glial fibrillary acidic protein (GFAP) expressed in astroglial cells in circadian rhythm under constant lighting conditions in mice. Journal of Neuroscience Research, 6o(2), 212-218.

Mrosovsky, N. (1999). Masking: History, definitions, and measurement. Chronobiology International, 16(4), 415-429.

Muñoz, E., M. Brewer, \& R. Baler (2006). Modulation of BMAL/CLOCK/E-Box complex activity by a CT-rich cis-acting element. Molecular and Cellular Endocrinology, 252(1), 74-81.

Panda, S., T.K. Sato, A.M. Castrucci, M.D. Rollag, W.J. DeGrip, J.B. Hogenesch, I. Provencio, \& S.A. Kay (2002). Melanopsin (Opn4) requirement for normal light-induced circadian phase shifting. Science, 298, 2213-2216.

Pati, A.K., \& S. Gupta (1994). Time estimation circadian rhythm in shift workers and diurnally active humans. Journal of Biosciences, 19(3), 325-330.

Pittendrigh, C.S. (1965). On the mechanism of the entrainment of a circadian rhythm by light cycles. In Aschoff, J., Circadian clocks. Amsterdam: Elsevier.

Plano, S.A., P.V. Agostino, H.O. de la Iglesia, \& D.A. Golombek (2012). cGMP-phosphodiesterase inhibition enhances photic responses and synchronization of the biological circadian clock in rodents. PLoS One, 7(5), e37121.

Preitner, N., F. Damiola, L. Lopez-Molina, J. Zakany, D. Duboule, U. Albrecht, \& U. Schibler (2002). The orphan nuclear receptor REV-ERBalpha controls circadian transcription within the positive limb of the mammalian circadian oscillator. Cell, $110,251-260$.

Refinetti, R. (1993). Laboratory instrumentation and computing: Comparison of six methods for the determination of the period of circadian rhythms. Physiology \& Behavior, 54(5), 869-875.

Reid, K.J. \& S.M. Abbott (2015). Jet lag and shift work disorder. Sleep Medicine Clinics, $10(4), 5^{23}-535$. 
Santhi, N., T.S. Horowitz, J.F. Duffy, \& C.A. Czeisler (2007). Acute sleep deprivation and circadian misalignment associated with transition onto the first night of work impairs visual selective attention. PLoS One, 2, e1233.

Sato, T.K., S. Panda, L.J. Miraglia, T.M. Reyes, R.D. Rudic, P. McNamara, K.A. Naik, G.A. FitzGerald, S.A. Kay, \& J.B. Hogenesch (2004). A functional genomics strategy reveals Rora as a component of the mammalian circadian clock. Neuron, 43, 527-537.

Scheer, F.A., M.F. Hilton, C.S. Mantzoros, \& S.A. Shea (2009). Adverse metabolic and cardiovascular consequences of circadian misalignment. Proceedings of the National Academy of Science USA, 106, 4453-4458.

Shurtleff, D., T.G. Raslear, \& L. Simmons (1990). Circadian variations in time perception in rats. Physiology \& Behavior, 47(5), 931-939.

Soshi, T., K. Kuriyama, S. Aritake, M. Enomoto, A. Hida, M. Tamura, Y. Kim, \& K. Mishima (2010). Sleep deprivation influences diurnal variation of human time perception with prefrontal activity change: A functional near-infrared spectroscopy study. PLoS One, 5 , e8395.

Sudo, M., K. Sasahara, T. Moriya, M. Akiyama, T. Hamada, \& S. Shibata (2003). Constant light housing attenuates circadian rhythms of mPer2 mRNA and mPER2 protein expression in the suprachiasmatic nucleus of mice. Neuroscience, 121(2), 493-499.

Tapia-Osorio, A., R. Salgado-Delgado, M. Angeles-Castellanos, \& C. Escobar (2013). Disruption of circadian rhythms due to chronic constant light leads to depressive and anxiety-like behaviors in the rat. Behavioural Brain Research, 252, 1-9.

Vilaplana, J., T. Cambras, \& A. Diez-Noguera (1997). Dissociation of motor activity circadian rhythm in rats after exposure to LD cycles of 4-h period. American Journal of Physiology-Regulatory, Integrative and Comparative Physiology, 272(1), R95-R102.

Welsh, D.K., \& S.A. Kay (2005). Bioluminescence imaging in living organisms. Current Opinion in Biotechnology, 16(1), 73-78.

Welsh, D.K., D.E. Logothetis, M. Meister, \& S.M. Reppert (1995). Individual neurons dissociated from rat suprachiasmatic nucleus express independently phased circadian firing rhythms. Neuron, 14, 697-706.

Wright, K.P. Jr., J.T. Hull, \& C.A. Czeisler (2002). Relationship between alertness, performance, and body temperature in humans. AmericanJournalof Physiology-Regulatory, Integrative and Comparative Physiology, 283(6), R1370-1377.

Yan, J., H. Wang, Y. Liu, \& C. Shao (2008). Analysis of gene regulatory networks in the mammalian circadian rhythm. PLoS Comput Biol, 4(10), e1000193.

Ye, R., C.P. Selby, Y.Y. Chiou, I. Ozkan-Daglivan, S. Gaddameedhi, \& A. Sancar (2014). Dual modes of CLOCK:BMAL inhibition mediates by Cryptochrome and Period proteins in the mammalian circadian clock. Genes \& development, 28(18), 1989-1998. Yoo, S.H., C.H. Ko, P.L. Lowrey, E.D. Buhr, E.J. Song, S. Chang, O.J. Yoo, S. Yamazaki, C. Lee, \& J.S. Takahashi (2005). A noncanonical E-box enhancer drives mouse 
Period2 circadian oscillations in vivo. Proceedings of the National Academy of Sciences USA, 102, 2608-2613.

Zar, J.H. (1999). Biostatistical Analysis. 4th edition. Prentice Hall, Upper Saddle River, New Jersey.

Zee, P.C., \& C.A. Goldstein (2010). Treatment of shift work disorder and jet lag. Current Treatment Options in Neurology, 12(5), 396-411.

Zhang, R., N.F. Lahens, H.I. Balance, M.E. Hughes, \& J.B. Hogenesch (2014). A circadian gene expression atlas in mammals: Implications for biology and medicine. Proceedings of the National Academy of Sciences USA, 111, 16219-16224.

Zielinski, T., A.M. Moore, E. Troup, K.J. Halliday \& A. J Millar (2014). Strengths and limitations of period estimation methods for circadian data. PLoS One, 9(5), eg6462. 